\title{
On the binding energies of antihydrogen formed by the interactions of antiprotons in cold positron plasmas
}

\author{
S Jonsell ${ }^{1, *}$ (1) and M Charlton ${ }^{2}$ (1) \\ ${ }^{1}$ Department of Physics, Stockholm University, SE-10691 Stockholm, Sweden \\ ${ }^{2}$ Department of Physics, College of Science, Swansea University, Swansea SA2 8PP, United Kingdom \\ E-mail: jonsell@fysik.su.se and m.charlton@swansea.ac.uk
}

Received 12 June 2020, revised 18 September 2020

Accepted for publication 25 November 2020

Published 05 February 2021

\begin{abstract}
The binding energies of antihydrogen atoms formed when antiprotons are mixed with positron plasmas having densities ranging from $10^{13}-10^{15} \mathrm{~m}^{-3}$, and at temperatures of 5-30 K, have been investigated using simulations. Major changes in the distribution of binding energies are observed, with more strongly bound states evident at the higher densities, and at lower temperatures. For deeper binding, the distribution of binding energies follows a power-law which is found to be strongly dependent upon plasma properties and the strength of the applied magnetic field. The underpinning role of collisions in determining the binding energies is explored.
\end{abstract}

Keywords: antihydrogen, antiprotons, binding energy, positron, plasma

(Some figures may appear in colour only in the online journal)

\section{Introduction}

The last two decades have witnessed remarkable progress in the development of experimental capabilities for the formation and study of neutral antimatter as antihydrogen, $\overline{\mathrm{H}}$, the antiproton-positron $\left(\bar{p}-\mathrm{e}^{+}\right)$bound state (for a review see, e.g., [1]). This has included the trapping [2-5] and stacking [6] of the anti-atom in magnetic minimum neutral atom traps, which has facilitated the first measurements of some of its properties [7-9]. This has culminated, to date, in observations of the first antihydrogen spectrum [10], the $1 S-2 S$ two-photon transition [11] and its lineshape [12], the $1 S-2 P$ Lyman- $\alpha$ transition [13] and fine structure of the first excited state of the anti-atom [14].

Following the first production of cold antihydrogen [15, 16], a number of groups undertook theoretical and compu-

* Author to whom any correspondence should be addressed.

(c) (i) Original content from this work may be used under the terms of the Creative Commons Attribution 3.0 licence. Any further distribution of this work must maintain attribution to the author(s) and the title of the work, journal citation and DOI. tational studies of $\overline{\mathrm{H}}$ formation via the three-body reaction,

$$
\mathrm{e}^{+}+\mathrm{e}^{+}+\bar{p} \rightarrow \overline{\mathrm{H}}+\mathrm{e}^{+},
$$

which, at typical experimental positron densities $\left(n_{\mathrm{e}}\right)$ and temperatures $\left(T_{\mathrm{e}}\right)$, is thought to be the dominant route for $\overline{\mathrm{H}}$ production [1]. This body of work has been reviewed by Robicheaux [17], and latterly we have contributed to this topic $[18,19]$ : recently with a study [20] involving simulations of the formation of $\overline{\mathrm{H}}$ at kinetic energies low enough to be held in the $0.54 \mathrm{~K}$ deep magnetic minimum trap used by the ALPHA experiment [21]. A key parameter is the $\overline{\mathrm{H}}$ binding energy, denoted as $E_{\mathrm{B}}$, which plays a crucial role in determining whether the anti-atoms created in the $\mathrm{e}^{+}$plasma survive to be detected and/or trapped. In our previous work it became clear that, under the conditions pertinent to current $\overline{\mathrm{H}}$ experimentation, the distribution of binding energies was dependent upon $n_{\mathrm{e}}$ and $T_{\mathrm{e}}$. This observation has prompted the detailed study reported here.

It is well known from theory (see the review in [22]) and experiment [23-25] that $\overline{\mathrm{H}}$ formed via reaction 1 is weakly bound, and strongly susceptible to influence from the ambient 
fields and from collisional effects: both have a role in determining the $E_{\mathrm{B}}$ distribution as the nascent anti-atoms leave the positron cloud. In the present work we have refined and extended the study reported in [20] to provide a detailed analysis of the behaviour of the $\overline{\mathrm{H}}$ binding energies in the range $T_{\mathrm{e}}=5-30 \mathrm{~K}$ and from $n_{\mathrm{e}}=10^{13}-10^{15} \mathrm{~m}^{-3}$, where we find that important qualitative and quantitative changes occur. We have also undertaken studies at applied magnetic fields, $B$, that are typical of those found in the ATRAP [16] and ALPHA [21] experiments.

The latter experiment has employed a base magnetic field of $1 \mathrm{~T}$ in a magnetic minimum $\overline{\mathrm{H}}$ trap. The radial increase in the trapping field [21] across the typical $(\sim \mathrm{mm})$ dimensions of their $\mathrm{e}^{+}$plasmas is negligible, so assuming a uniform $1 \mathrm{~T}$ field in the simulations is justified. ALPHA's $\mathrm{e}^{+}$plasma densities are typically in the range $5-10 \times 10^{13} \mathrm{~m}^{-3}$, but there is the capability to increase this if necessary. Recent ALPHA work (see e.g., [6]) has employed $\mathrm{e}^{+} \mathrm{s}$ with $T_{\mathrm{e}}$ in the range $15-20 \mathrm{~K}$, and the ability to reduce this to $5-10 \mathrm{~K}$ using active cooling via interaction with laser-cooled beryllium ions [26, 27] is currently being pursued $[28,29]$. ATRAP's original work on state analysis [23] was performed at $5.4 \mathrm{~T}$, and though there are no quoted values for $n_{\mathrm{e}}$ and $T_{\mathrm{e}}$ we can infer from their previous work on $\bar{p}$ cooling by positrons [30] (though performed at the slightly higher magnetic field of $6 \mathrm{~T}$ ) that the expected $T_{\mathrm{e}}$ is close to their trap ambient at $4.2 \mathrm{~K}$ and that $n_{\mathrm{e}}$ is around $7 \times 10^{12} \mathrm{~m}^{-3}$.

We compare the results of our work to the experimental data and accompanying analysis from ATRAP [23-25] and to the theoretical analyses of Bass and Dubin [31]. Both set of studies revealed that, at the higher values of $E_{\mathrm{B}}$, the distributions can be described by power laws (essentially as a function of $E_{\mathrm{B}} / k_{\mathrm{B}} T_{\mathrm{e}}$, with $k_{\mathrm{B}}$ being Boltzmann's constant), and we further elucidate this behaviour. Our simulations complement and augment this previous work and include full motion of both the $\mathrm{e}^{+}$and the $\bar{p}$ and, as described above, an exploration across the range of $n_{\mathrm{e}}$ and $T_{\mathrm{e}}$ of most relevance to experiment. The work of Bass and Dubin treated stationary antiprotons only, which fails to encapsulate important cross-field transport physics that contributes to $\overline{\mathrm{H}}$ production [19], and was applied to conditions of high magnetisation, corresponding to $\chi \ll 1$, with

$$
\chi=\frac{r_{\mathrm{c}}}{b}=\frac{\bar{v}}{b \omega_{\mathrm{c}}}
$$

which is not strictly applicable to all experimental situations. Here the Larmor radius is $r_{\mathrm{c}}=m \bar{v} / e B$, where the average thermal speed is taken as $\bar{v}=\omega_{\mathrm{c}} r_{\mathrm{c}}=\sqrt{k_{\mathrm{B}} T_{\mathrm{e}} / m}$. The distance of closest approach is given by $b=e^{2} / 4 \pi \epsilon_{0} k_{\mathrm{B}} T_{\mathrm{e}}$, with the cyclotron angular frequency as $\omega_{\mathrm{c}}=e B / \mathrm{m}$ : $e$ and $\epsilon_{0}$ have their usual meanings and $m$ is the positron rest mass. Numerically, $\chi \approx 1.35 \times 10^{-3} T_{\mathrm{e}}^{3 / 2} / B$. Simulations similar to ours (though still assuming a stationary antiproton), but with a focus on the cascade and resulting yield of ground-state atoms have also been performed by Radics et al [32,33].

In the remainder of the article we briefly introduce the simulations and the methods used (section 2), present and discuss the main findings (section 3 ) and conclude in section 4.

\section{Simulation details}

Comprehensive accounts of the methodology used in our simulations have been given elsewhere [18-20], such that we present a brief survey here with emphasis on the details most pertinent for the discussion of the $\overline{\mathrm{H}}$ binding energies. Classical equations of motion (as pointed out in [17, 19], quantum mechanical effects are only expected to play a role at much smaller distances, characteristic of much more deeply bound $\overline{\mathrm{H}}$ ) were used to calculate the antiparticle trajectories in an axial (conventionally the $z$-direction) magnetic field of $B=1$ or $5.4 \mathrm{~T}$ in the presence of the self electric field, $\mathbf{E}$, of the $\mathrm{e}^{+}$cloud. The latter is given by $\mathbf{E}=n_{\mathrm{e}} e \boldsymbol{\rho} / 2 \epsilon_{0}=E_{\rho} \rho \hat{\boldsymbol{\rho}}$ where $\boldsymbol{\rho}=\rho \hat{\boldsymbol{\rho}}$ is the radial coordinate with the origin at the trap axis. The combination of fields results in a rotation of both $\mathrm{e}^{+}$and $\bar{p}$ about the axis with a speed to first order given by, $v_{\mathrm{D}}=E_{\rho} \rho / B=n_{\mathrm{e}} e \rho / 2 \epsilon_{0} B$.

The $\bar{p}$ trajectories were initialised from a thermal distribution at $T_{\mathrm{e}}$ with the $\mathrm{e}^{+}$plasma taken, for simplicity, as an infinitely long cylinder of $1 \mathrm{~mm}$ radius. This approximation does not affect the outcome of the simulation, as detailed elsewhere [20]. In that work, the fraction of the formed $\overline{\mathrm{H}}$ which was deemed to be trappable was deduced by applying several criteria. Whilst conditions which pertain strictly to being held in the trap (i.e., those for kinetic energy and magnetic moment) can be relaxed here, first and foremost, the anti-atoms must be able to survive their environment and emerge from the $\mathrm{e}^{+}$ plasma in a stably bound state. It therefore proved crucial that such states could be separated from those instances in which a $\bar{p}$ left the plasma with a $\mathrm{e}^{+}$nearby, but unbound.

To achieve this an operational definition of a stable $\overline{\mathrm{H}}$ atom was developed. First, the $\bar{p}-\mathrm{e}^{+}$pair should remain together for a duration of $1 \mu \mathrm{s}$ in the magnetic field and electric fields present outside the cylindrical plasma $\left(\propto \rho^{-1}\right)$. Secondly, to mimic the electric fields of a typical Penning trap used to hold the antiparticles, an axial electric field of $10 \mathrm{Vcm}^{-1}$ was applied during the $1 \mu \mathrm{s}$ flight time. Using guidance from [34], a crude estimate shows that $\overline{\mathrm{H}}$ with $E_{\mathrm{B}}$ lower than about $9 \sqrt{E} \mathrm{~K}$, with $E$ in $\mathrm{Vcm}^{-1}$, corresponding to $\sim 28 \mathrm{~K}$, will be ionised by the electric field. Though this value for the electric field is somewhat arbitrary, tests reveal that while the number of bound $\overline{\mathrm{H}}$ with $E_{\mathrm{B}}<9 \sqrt{E} \mathrm{~K}$ changes with the magnitude of the applied field, the distribution of more deeply bound anti-atoms is not affected.

Our simulation uses the unscreened Coulomb potential, thus neglecting any plasma effects. However, screening occurs on length scales comparable to the Debye length $\lambda_{\mathrm{D}}=\sqrt{\frac{k_{\mathrm{B}} T_{\mathrm{e}} \epsilon_{0}}{e^{2} n_{\mathrm{e}}}}$, which for our parameters ranges from $5 \mu \mathrm{m}$ to $120 \mu \mathrm{m}$, whereas the length scale relevant for antihydrogen formation is set by the radius of the anti-atom. Assuming an $\overline{\mathrm{H}}$ bound by at least the ambient temperature, this length varies from $0.3 \mu \mathrm{m}$ to $1.7 \mu \mathrm{m}$. Since the relevant dynamics for anti-atom formation occurs on scales on order of magnitude shorter than Debye-length we do not expect significant changes, though we cannot rule out that at our lowest temperatures and largest density some correction due to this effect could occur. 


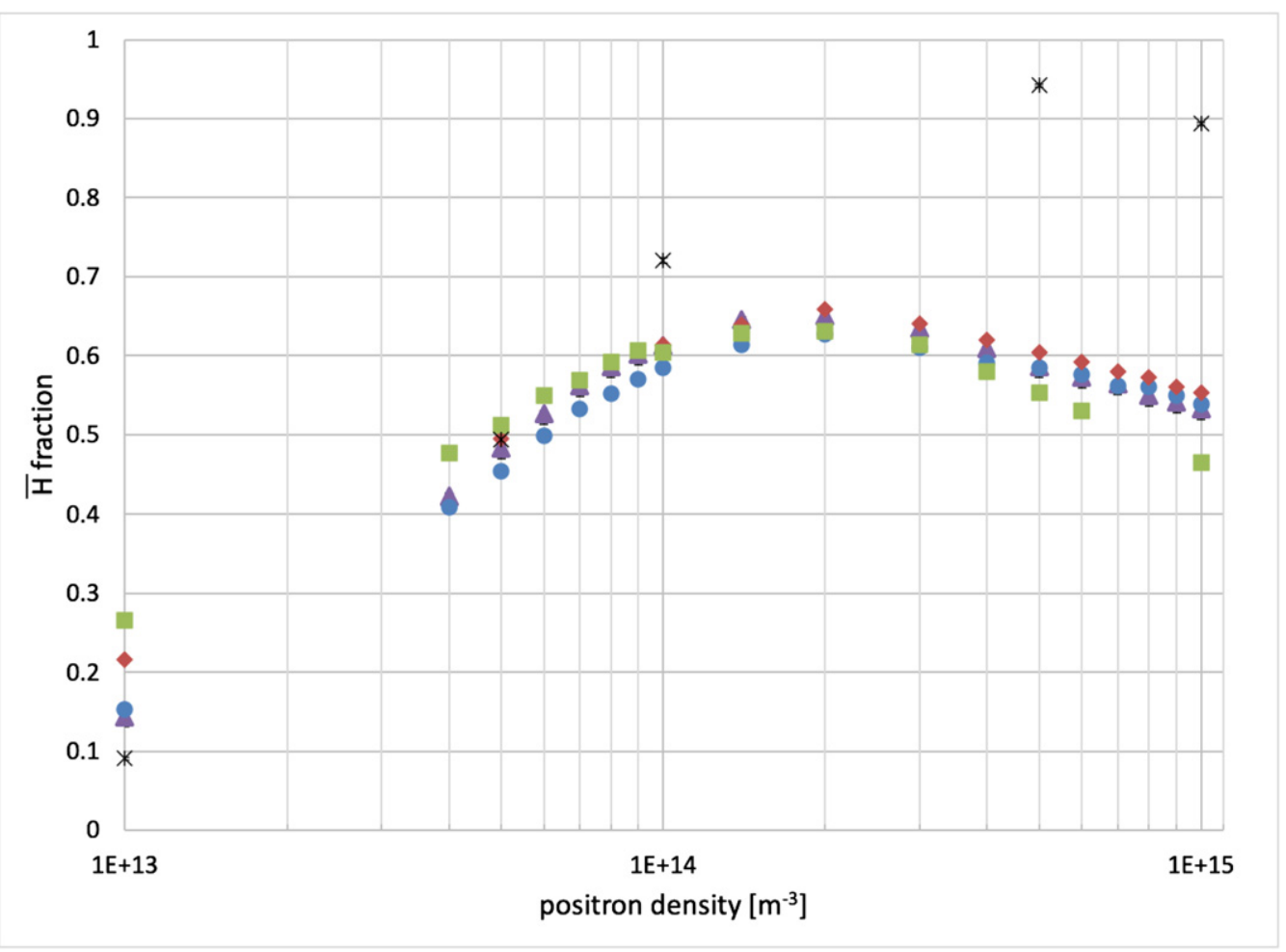

Figure 1. The fraction of antiprotons resulting in stable $\overline{\mathrm{H}}$ atoms (see text) at various plasma densities, $n_{\mathrm{e}}$. At $B=1 \mathrm{~T}$ the $T_{\mathrm{e}}$ data are shown as $5 \mathrm{~K}$ (purple triangles), $10 \mathrm{~K}$ (blue circles), $15 \mathrm{~K}$ (red diamonds) and $30 \mathrm{~K}$ (green squares), with the last three reproduced from [20]. For comparison results for $B=5.4 \mathrm{~T}$ and $T_{\mathrm{e}}=5 \mathrm{~K}$ are shown (black stars). Note that all data correspond to injection of the $\bar{p}$ on the axis of the trap, i.e. $\rho_{0}=0$.

The fraction of $\bar{p} \mathrm{~s}$ resulting in $\overline{\mathrm{H}}$ which survives this treatment was referred to as the $\overline{\mathrm{H}}$-fraction, $f_{\overline{\mathrm{H}}}$, in [20], and it is from this ensemble that the data we present in section 3 is drawn. We note, following [20], that radiative de-excitation processes (which will eventually lead to $\overline{\mathrm{H}}$ in the ground state) are not included, as these are not important during the first stages of $\overline{\mathrm{H}}$ formation $[31,32]$ considered here.

As will become evident in section 3 , collisional effects play an important role in increasing $E_{\mathrm{B}}$ as the $\overline{\mathrm{H}}$ moves through the $\mathrm{e}^{+}$plasma. This is particularly so as $T_{\mathrm{e}}$ is lowered, where the anti-atom is initially formed in very high Rydberg-like states. However, in the cases when the $\overline{\mathrm{H}}$ reaches very deep binding, numerical problems arise due to the divergence of the Coulomb potential at short $\bar{p}-\mathrm{e}^{+}$separations. To ensure energy conservation this necessitates the use of vanishingly small time steps, which cause the simulation to slow dramatically, and sometimes to come to a complete halt. This is mainly a problem at the higher $n_{\mathrm{e}}$, where the $\overline{\mathrm{H}}$ reach deeper binding energies. In order to remove this problem, we (i) introduced a softening of the Coulomb potential and (ii) froze the internal state of any antihydrogen with binding energy $E_{\mathrm{B}}>300 \mathrm{~K}$. Our softened Coulomb potential has the form

$$
V(r)=-\frac{e^{2}}{4 \pi \epsilon_{0}} \frac{1}{\sqrt{r^{2}+r_{0}^{2}}}
$$

where $r_{0}$ is a constant that removes the singularity as $r \rightarrow 0$. After testing, we determined that our results were sta- ble using $r_{0}=2 \times 10^{-8} \mathrm{~m}$, corresponding to the radius of an $\overline{\mathrm{H}}$ bound by $420 \mathrm{~K}$, thus below the deepest binding energy in our simulation.

Therefore, our results for binding energies above $300 \mathrm{~K}$ cannot be trusted, but this should, for all temperatures simulated, be safely above the so-called 'bottleneck' at a few $k_{\mathrm{B}} T_{\mathrm{e}}{ }^{3}$

\section{Results and discussion}

Our previous work [18-20] revealed that the observed (and trapped) $\overline{\mathrm{H}}$ is the result of many cycles of formation and collisional break-up (by the reverse of reaction 1, i.e., $\mathrm{e}^{+}+\overline{\mathrm{H}} \rightarrow$ $\mathrm{e}^{+}+\mathrm{e}^{+}+\bar{p}$ ). This cycling effect results in radial transport of the bound $\bar{p} \mathrm{~s}$ in the $\mathrm{e}^{+}$plasma, and is a major determinant of the final $\overline{\mathrm{H}}$ binding energy. Figure 1 reproduces the data for $f_{\overline{\mathrm{H}}}$ from [20] at $1 \mathrm{~T}$, and adds new results at $T_{\mathrm{e}}=5 \mathrm{~K}$ and at that temperature at $5.4 \mathrm{~T}$, in order to frame the discussion to follow. From this figure it is clear that there are notable trends with both $n_{\mathrm{e}}$ and $T_{\mathrm{e}}$ with, in particular, a change of behaviour,

\footnotetext{
${ }^{3}$ The bottleneck is typically taken to be a binding energy of $4-6 k_{\mathrm{B}} T_{\mathrm{e}}$. When $E_{\mathrm{B}}$ is higher than this, there is very little probability of re-ionisation of the nascent $\overline{\mathrm{H}}$ in a subsequent collision, and the anti-atom will cascade to deeper binding, eventually reaching the ground state. The energy range of a few $k_{\mathrm{B}} T_{\mathrm{e}}$ on either side of the (field-free) ionisation limit is usually thought of as comprising a thermal bath of positrons, from which they trickle to binding above the energy of the one-way bottleneck via collision. Further details can be found elsewhere [35-38].
} 

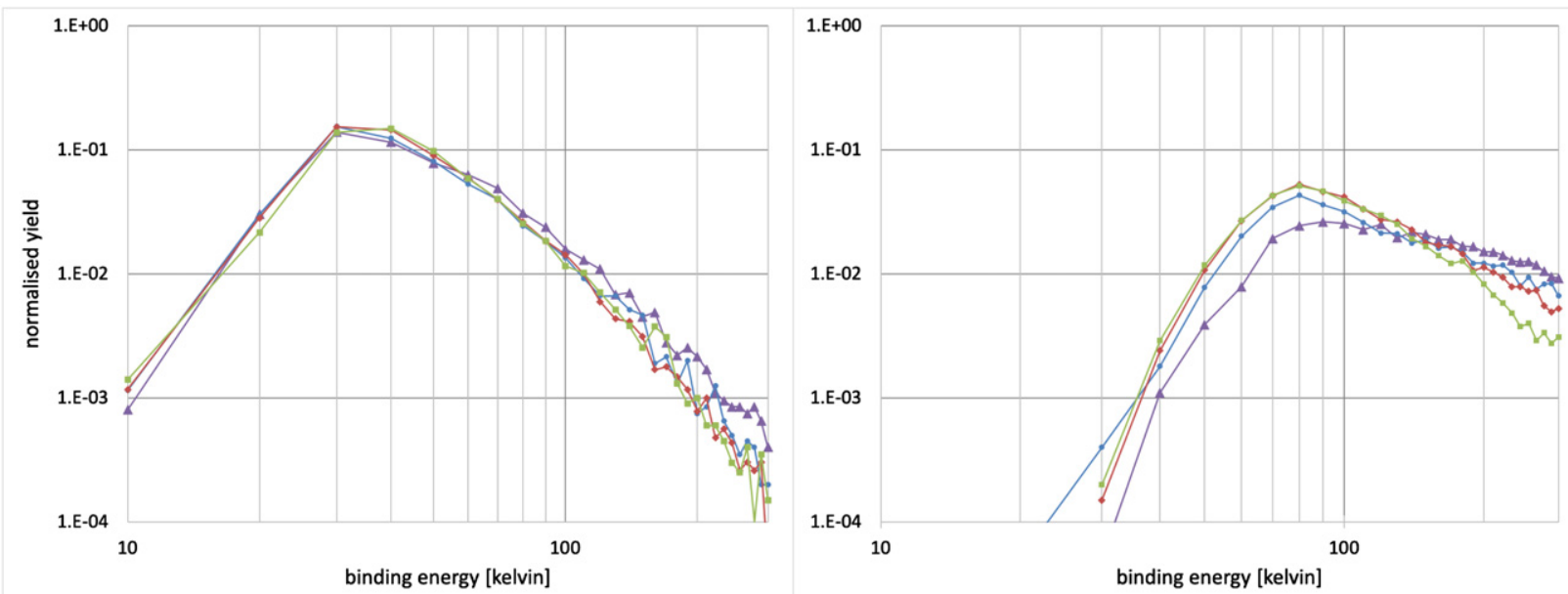

Figure 2. The binding energy distributions, for $B=1 \mathrm{~T}$, normalised per simulated antiproton, for stable $\overline{\mathrm{H}}$ at $n_{\mathrm{e}}=10^{14} \mathrm{~m}^{-3}(\mathrm{left}$ panel) and $n_{\mathrm{e}}=10^{15} \mathrm{~m}^{-3}$ (right panel) for $T_{\mathrm{e}}=5,10,15$ and $30 \mathrm{~K}$, with the temperatures colour-coded as in figure 1 . The lines are drawn to guide the eye and are not fits to the data.

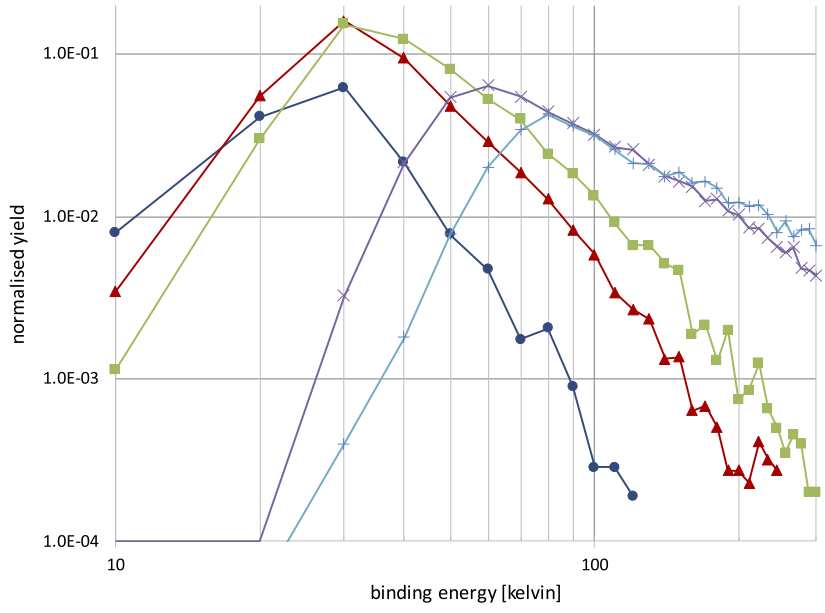

Figure 3. The binding energy distributions, for $B=1 \mathrm{~T}$, normalised per simulated antiproton, for stable $\overline{\mathrm{H}}$ at $T_{\mathrm{e}}=10 \mathrm{~K}$ and at various $n_{\mathrm{e}}$. The data are: $n_{\mathrm{e}}=1 \times 10^{13} \mathrm{~m}^{-3}$ (dark blue circles); $n_{\mathrm{e}}=5 \times 10^{13} \mathrm{~m}^{-3}$ (red triangles); $n_{\mathrm{e}}=1 \times 10^{14} \mathrm{~m}^{-3}$ (green squares); $n_{\mathrm{e}}=5 \times 10^{14} \mathrm{~m}^{-3}$ (purple crosses); $n_{\mathrm{e}}=1 \times 10^{15} \mathrm{~m}^{-3}$ (light blue plus signs). The lines are drawn to guide the eye and are not fits to the data.

dependent upon the magnitude of $B$, in the density region from $10^{14}-10^{15} \mathrm{~m}^{-3}$ as $n_{\mathrm{e}}$ is varied. This effect is accompanied by major changes in the $\overline{\mathrm{H}} E_{\mathrm{B}}$ distributions, which then also display a marked dependence upon $T_{\mathrm{e}}$. These phenomena can be traced back to changes in the mean radial position of origin of the $\overline{\mathrm{H}}$ and as we shall see, at the higher densities, the anti-atoms are predominantly formed close to the trap axis, thus enhancing the probability of achieving deeper binding before leaving the $\mathrm{e}^{+}$plasma.

Examining figure $1, f_{\overline{\mathrm{H}}}$ rises steadily when $n_{\mathrm{e}} \lesssim 10^{14} \mathrm{~m}^{-3}$ at all $T_{\mathrm{e}}$, and at both values of $B$. Interestingly, at the lower $n_{\mathrm{e}}$, $f_{\overline{\mathrm{H}}}$ is higher as $T_{\mathrm{e}}$ is raised, though the trend seems to cross over with increasing density. At $B=1 \mathrm{~T}$ the $f_{\overline{\mathrm{H}}}$ values are broadly comparable across the $T_{\mathrm{e}}$ range. This behaviour is caused by the many $\bar{p}$ interactions that take place in the plasma which
Table 1. Power-law exponents $(n)$ of the binding-energy distributions for different parameters, and for a starting radius $\rho_{0}=0 \mathrm{~mm}$. The power laws were fitted to the deep part of the binding energy distributions. Because of the differences in the distributions it was not possible to use the same fit interval for all cases.

\begin{tabular}{lcccccc}
\hline & & \multicolumn{5}{c}{$n_{\mathrm{e}}\left(\mathrm{m}^{-3}\right)$} \\
\cline { 3 - 7 }$B(\mathrm{~T})$ & $T_{\mathrm{e}}(\mathrm{K})$ & $10^{13}$ & $5 \times 10^{13}$ & $10^{14}$ & $5 \times 10^{14}$ & $10^{15}$ \\
\hline 5.4 & 5 & 5.6 & 5 & 4.8 & 3.6 & 2.8 \\
1 & 5 & 4.5 & 3.7 & 3.4 & 1.9 & 1.0 \\
1 & 10 & 4.2 & 3.7 & 3.5 & 1.8 & 1.2 \\
1 & 15 & 4.3 & 3.9 & 3.9 & 2.6 & 1.9 \\
1 & 30 & 4.1 & 3.6 & 4.3 & 2.8 & 2.7 \\
\hline
\end{tabular}

eventually result in similar antihydrogen fractions, despite the rate of reaction 1 being expected to fall (in equilibrium and in the limit when $\chi \rightarrow \infty$ ) by a factor of $6^{4.5} \approx 3170$ as $T_{\mathrm{e}}$ is raised. Though the timescales are longer at higher $T_{\mathrm{e}}$, the underlying physics ensures a similar outcome. At higher $n_{\mathrm{e}}$, there is a marked change of behaviour with $f_{\overline{\mathrm{H}}}$ falling in each case, but more rapidly for higher $T_{\mathrm{e}}$. There is also a striking difference between the $5 \mathrm{~K}$ data at different magnetic fields, with those at $B=5.4 \mathrm{~T}$ displaying a larger slope at low densities, turning over at a significantly higher density and reaching a peak at a higher value of $f_{\overline{\mathrm{H}}}$.

Figure 2 shows the normalised $E_{\mathrm{B}}$ distributions at $n_{\mathrm{e}}=10^{14} \mathrm{~m}^{-3}$ and $n_{\mathrm{e}}=10^{15} \mathrm{~m}^{-3}$ at the various $T_{\mathrm{e}}$ (all at a magnetic field of $1 \mathrm{~T}$ ), whilst figures 3 and 4 present the data for various $n_{\mathrm{e}}$ at fixed magnetic field and temperature. The distributions have some common features, rising at low binding to a density-dependent maximum in the 10's of K region. This is followed by a decline at higher binding which takes the approximate form of a power-law dependence (i.e., $E_{\mathrm{B}}^{n}$, with exponent, $n)$ in a manner similar to the observations of ATRAP [25] and Bass and Dubin [31], though here we identify significant changes in behaviour with $T_{\mathrm{e}}$ and in particular $n_{\mathrm{e}}$. Fitted 

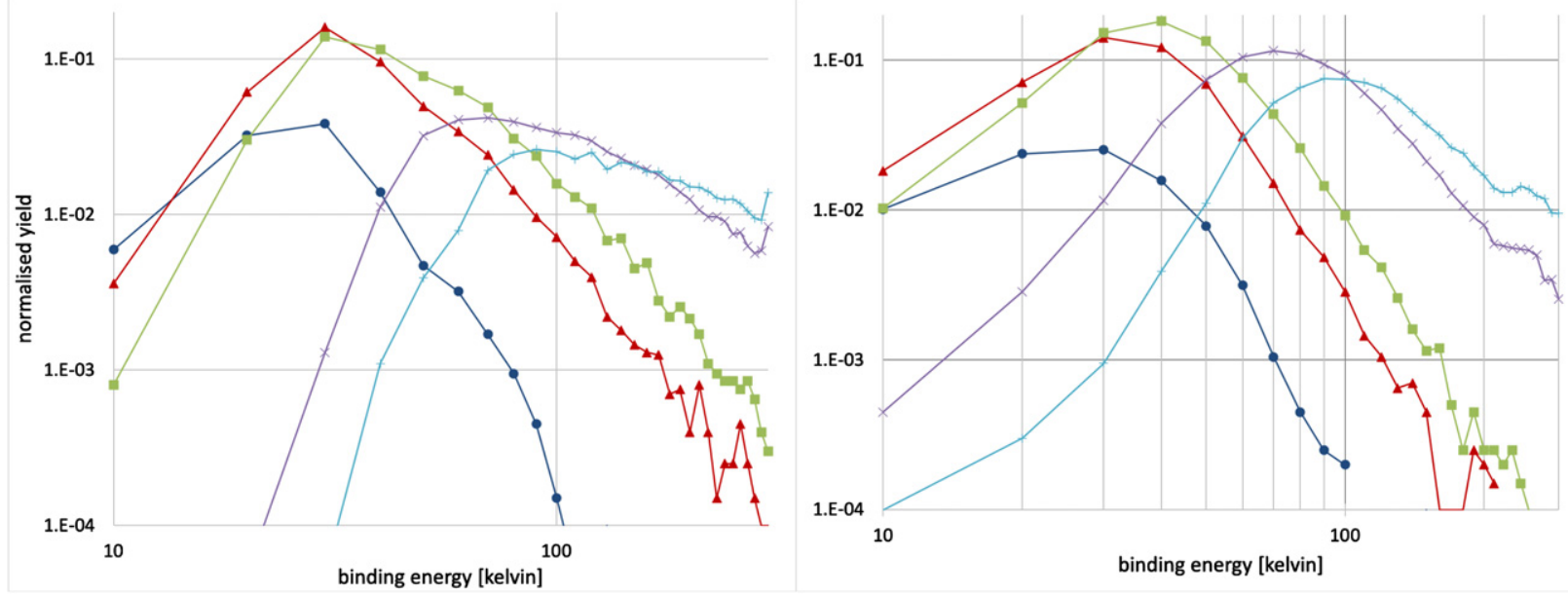

Figure 4. Same as figure 3, except $T_{\mathrm{e}}=5 \mathrm{~K}$. The values for the magnetic fields are $B=1 \mathrm{~T}$ (left panel) and $B=5.4 \mathrm{~T}$ (right panel).

values for the power-law exponents at the higher $E_{\mathrm{B}}$ for various $\mathrm{e}^{+}$plasma parameters are given in table 1 .

Turning first to the left panel of figure 2, despite the factor of six change in $T_{\mathrm{e}}$, the form of the four curves is remarkably similar, with some states bound by more than $100 \mathrm{~K}$ in evidence. The binding energies rise from close to the $28 \mathrm{~K}$ cut-off imposed in the simulations through the axial electric field outside the plasma (though there are some more weakly bound $\overline{\mathrm{H}}$ that survive in each case) and peak for all $T_{\mathrm{e}}$ at around $30-40 \mathrm{~K}$. This corresponds to around 3-4 meV, or a principal quantum number of just over 60, though the latter is ill-defined for such states in the high magnetic field.

At the higher $E_{\mathrm{B}}$ the distributions are very similar, which is reflected by the values for $n$ summarised in table 1 , though there is a trend towards deeper binding (i.e., lower values of $n)$ as $T_{\mathrm{e}}$ is lowered. We note that while our simulations are similar to those in [25] they differ from [31] both in that we include the motion of the $\bar{p} \mathrm{~s}$ (as noted earlier) and since we simulate the injection of a fixed population of $\bar{p} \mathrm{~s}$, which over time is depleted as $\overline{\mathrm{H}} \mathrm{s}$ are formed or the antiprotons leave the plasma. It is therefore not surprizing that we do not obtain a steady-state distribution as a function of $E_{\mathrm{B}} / k_{\mathrm{B}} T$, which was found in [31].

The corresponding distributions at $n_{\mathrm{e}}=10^{15} \mathrm{~m}^{-3}$ are shown in the right panel of figure 2 . Whilst the form of the curves at the different $T_{\mathrm{e}}$ are similar, there are significant differences, and there is a marked change in the distributions when compared to the data at the lower density. In each case the number of stable anti-atoms increases after about $35 \mathrm{~K}$, to reach a broad maximum around $80 \mathrm{~K}$, the location of which is nearly independent of $T_{\mathrm{e}}$. Binding energies now extend out to $300 \mathrm{~K}$ and above for all $T_{\mathrm{e}}$ and there is a notable and systematic increase in the yield at higher $E_{\mathrm{B}}$ as $T_{\mathrm{e}}$ is reduced. The resulting power-law exponents given in table 1 are lower than at $10^{14} \mathrm{~m}^{-3}$, approaching unity for $T_{\mathrm{e}}$ in the range $5-10 \mathrm{~K}$.

The $\overline{\mathrm{H}}$ binding energy on exit from the plasma will depend mainly upon the number and type of collisions the anti-atom undergoes. Whilst collisions can break up the nascent $\overline{\mathrm{H}}$, they will also provide the drive towards deeper binding as the $\mathrm{e}^{+}$ can efficiently remove the excess energy. Thus, the binding energy is expected to increase with $n_{\mathrm{e}}$, and this is roughly in accord with the simulation data. However, the distributions in figure 2 reveal a more complex picture, as $T_{\mathrm{e}}$ clearly has a major influence as $n_{\mathrm{e}}$ is increased. The dependence upon $T_{\mathrm{e}}$ is complicated by the fact that there are two competing effects. On one hand larger energy exchanges will occur at higher $T_{\mathrm{e}}$, and larger positron speeds give higher collision rates, though this is offset since $\overline{\mathrm{H}}$ formation is much less likely, leading to fewer $\mathrm{e}^{+}-\overline{\mathrm{H}}$ collisions, and less likelihood of stable $\overline{\mathrm{H}}$. It is the cumulative effect of collisions (see e.g., [31]) which leads to stability, as $E_{\mathrm{B}}$ becomes larger than the bottleneck energy at a few $k_{\mathrm{B}} T_{\mathrm{e}}$, below which the probability of ionisation is negligible, and the anti-atom will survive.

To illuminate further, figure 3 presents binding energy distributions in the range $n_{\mathrm{e}}=10^{13}-10^{15} \mathrm{~m}^{-3}$ at $T_{\mathrm{e}}=10 \mathrm{~K}$ and $B=1 \mathrm{~T}$, showing the systematic shift towards higher $E_{\mathrm{B}}$ as $n_{\mathrm{e}}$ is raised above $\approx 10^{14} \mathrm{~m}^{-3}$. The two highest densities, $n_{\mathrm{e}}=5 \times 10^{14}$ and $10^{15} \mathrm{~m}^{-3}$ have similar distributions at high binding energies, indicating that they have undergone enough collisions to reach a steady state.

Again, the tails of the distributions towards larger binding energies have roughly a power-law dependence, as was found in [31]. The corresponding data at $T_{\mathrm{e}}=5 \mathrm{~K}$ (figure 4) show similar features for both our standard value of the magnetic field $B=1 \mathrm{~T}$ and the larger field $B=5.4 \mathrm{~T}$ used in [23]. The depression of the yield evident at low $E_{\mathrm{B}}$ for the two highest values of $n_{\mathrm{e}}$ is discussed further below, but we note here that the effect is reduced at the larger magnetic field.

Table 1 presents the fitted power-law exponents across the parameter range of our investigation. At the larger magnetic field and low $T_{\mathrm{e}}$ characteristic of the ATRAP work, we find somewhat larger values for $n$ (thus fewer anti-atoms at deep binding) when compared to their equivalent at $1 \mathrm{~T}$. This is consistent with the increased degree of magnetisation of the $\mathrm{e}^{+}$ plasma, which inhibits collisions due to pinning of the charged particles on the field lines [39]: note that at this combination of $B$ and $T_{\mathrm{e}}, \chi \approx 2.2 \times 10^{-3}$. At the lower densities investigated we find $n \sim 5-6$, which is roughly consistent with value of 5 from the analysis of Pohl and co-workers [25] of the ATRAP field-ionisation binding energy data [23]. Recall from section 1 
Table 2. Power-law exponents ( $n$ ) of the binding-energy distributions for

$B=1 \mathrm{~T}, n_{\mathrm{e}}=10^{14} \mathrm{~m}^{-3}, n_{\mathrm{e}}=10^{15} \mathrm{~m}^{-3}$ and different temperatures and starting radii, $\rho_{0}$.

\begin{tabular}{lcccccccccc}
\hline & & \multicolumn{10}{c}{$\rho_{0}(\mathrm{~mm})$} \\
\cline { 3 - 10 }$n_{\mathrm{e}}\left(\mathrm{m}^{-3}\right)$ & $T_{\mathrm{e}}(\mathrm{K})$ & 0 & 0.01 & 0.02 & 0.05 & 0.1 & 0.2 & 0.3 & 0.4 & 0.5 \\
\hline$n_{\mathrm{e}}=10^{14}$ & 10 & 3.9 & & & & 3.7 & 3.7 & 3.8 & 4.0 & 3.7 \\
$n_{\mathrm{e}}=10^{15}$ & 5 & 1.0 & 1.1 & 1.3 & 2.2 & 3.9 & 4.4 & 4.3 & 4.8 & 4.4 \\
$n_{\mathrm{e}}=10^{15}$ & 10 & 1.2 & 1.3 & 1.4 & 2.2 & 3.6 & 4.6 & 4.4 & 4.7 & 4.6 \\
$n_{\mathrm{e}}=10^{15}$ & 15 & 1.9 & 1.9 & 1.9 & 2.3 & 3.2 & 4.1 & 4.5 & 5.4 & 4.8 \\
$n_{\mathrm{e}}=10^{15}$ & 30 & 2.7 & 2.9 & 2.8 & 2.7 & 3.3 & 4.1 & 4.7 & 5.0 & 5.5 \\
\hline
\end{tabular}

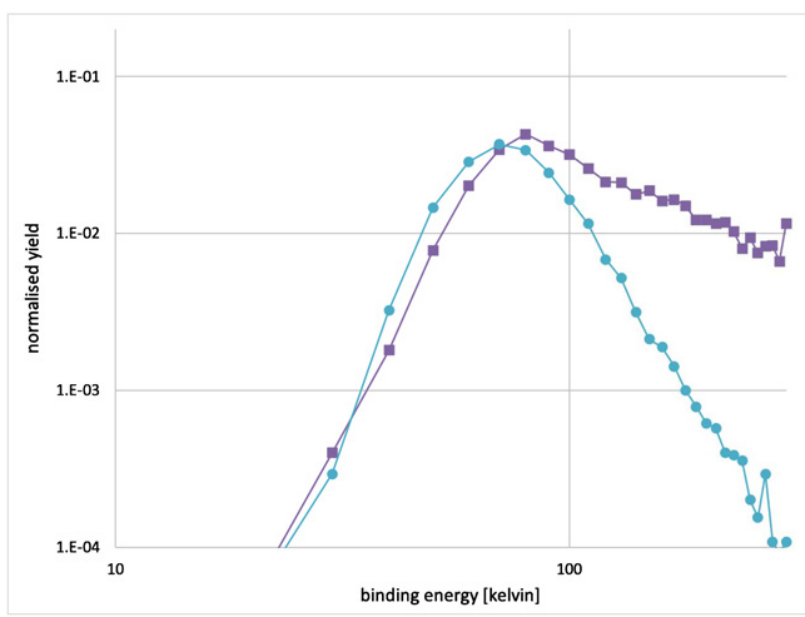

Figure 5. Distribution of binding energies for $B=1 \mathrm{~T}, T=10 \mathrm{~K}$, $n_{\mathrm{e}}=10^{15} \mathrm{~m}^{-3}$ and $\rho_{0}=0 \mathrm{~mm}$ (purple squares) and $0.5 \mathrm{~mm}$ (blue circles).

that we presume that the positron density employed by ATRAP was around $10^{13} \mathrm{~m}^{-3}$ at a temperature close to $4 \mathrm{~K}$.

Our analysis implies that the time spent by the $\overline{\mathrm{H}}$ inside the finite radius positron plasma plays a role in determining the distribution of binding energies, and hence the variation in $n$. Bass and Dubin [31] analysed the time dependence of their $E_{\mathrm{B}}$ distributions and extracted exponents with similar values to those found here. For instance at $\chi=0.005$ they found $n \simeq 5.5$, which rose to a value of 8 for $\chi=0$ (corresponding to weaker binding in an infinite magnetic field).

At $B=1 \mathrm{~T}$ we find $n \simeq 4$ for densities below $10^{14} \mathrm{~m}^{-3}$, with lower temperatures giving somewhat larger exponents. At higher densities the picture changes drastically, with a sharp drop in $n$, which is most pronounced at low $T_{\mathrm{e}}$. From previous work we know that at these densities it is important to take the drift motion of the particles into account [20]. The velocity of this motion grows with the distance between the particle and the axis of the trap (see below). In order to investigate this effect, we performed further simulations of how the binding energy distribution depends on the trap radius $\rho_{0}$ at which the antiproton is injected. These results are summarised in table 2 .

As the injection radius is increased, the high-density exponents approach values similar to, or even higher than, those at lower densities. This effect is illustrated by the example given in figure 5. When the antiprotons are injected on the trap axis $\left(\rho_{0}=0 \mathrm{~mm}\right)$ there is a component of deeply bound anti-atoms, which is not present if injection occurs far from the trap axis $\left(\rho_{0}=0.5 \mathrm{~mm}\right)$. Below we give a plausible explanation for this behaviour.

Though $\overline{\mathrm{H}}$ is formed by the reaction 1 at all radii for both densities, at the higher density and larger radii the initial unstable state is much less likely to develop into a stable state. We attribute this effect to the mismatch in the drift velocities of positrons and antiprotons. It is well known that trapped particles with charge $q$ and mass $m$ rotate around the axis of the trap with an angular frequency given by

$$
\Omega_{-}=-\frac{1}{2}\left(\omega_{\mathrm{c}}+\sqrt{\omega_{\mathrm{c}}^{2}-\frac{4 q E_{\rho}}{m}}\right),
$$

where $\omega_{\mathrm{c}}=q B / m$ is the cyclotron frequency and $E_{\rho}$ was defined in section 2 and is due to the radial electric field generated by the charge of the positron plasma. To first order, this gives a rotation frequency $\omega_{-} \sim E_{\rho} / B$, and thus the drift speed $v_{\mathrm{D}}=n_{\mathrm{e}} e \rho / 2 \epsilon_{0} B$, as introduced in section 2 , which is the same for positrons and antiprotons. At large densities, though, the second-order correction to this speed becomes important. This term has the form $\Delta v_{\mathrm{D}}=m E_{\rho}^{2} \rho / q B^{3}=m n_{\mathrm{e}}^{2} e^{2} \rho / 4 q \epsilon_{0}^{2} B^{3}$, and thus grows linearly with mass and trap radius, and quadratically with plasma density. At the densities considered here for $B=1 \mathrm{~T}$, the effect is negligible for the lighter positron, but for the antiproton it grows from a correction of less than $1 \%$ at $n_{\mathrm{e}}=10^{14} \mathrm{~m}^{-3}$ to around $10 \%$ at $n_{\mathrm{e}}=10^{15} \mathrm{~m}^{-3}$.

In the reference frame of the antiproton, this effect appears as a fictitious force on the positron. This force is too weak to have any impact on tightly bound antihydrogen atoms, but in its initial loosely bound state it is enough to rip the nascent antiatom apart, thus preventing it from stabilising. The exception is when formation occurs close to the axis of the trap, where the correction is vanishing. This explains why the gap in the distribution of binding energies in figure 3 at high densities extends beyond the $\sim 28 \mathrm{~K}$ expected from field ionisation ${ }^{4}$. Any surviving antihydrogen must have been formed close to the axis, in a state with binding energy large enough to traverse the entire plasma without ionising in the large number of collisions with positrons. Similarly, the higher low- $E_{\mathrm{B}}$ yields

${ }^{4}$ This mechanism for ionisation of loosely bound antihydrogen was observed already in the simulations reported in [18], though the impact on the distribution of binding energies was not realised at the time. Instead the increased gap at high densities was ascribed, we believe incorrectly, to direct field ionisation due to the higher electric field from the plasma. 


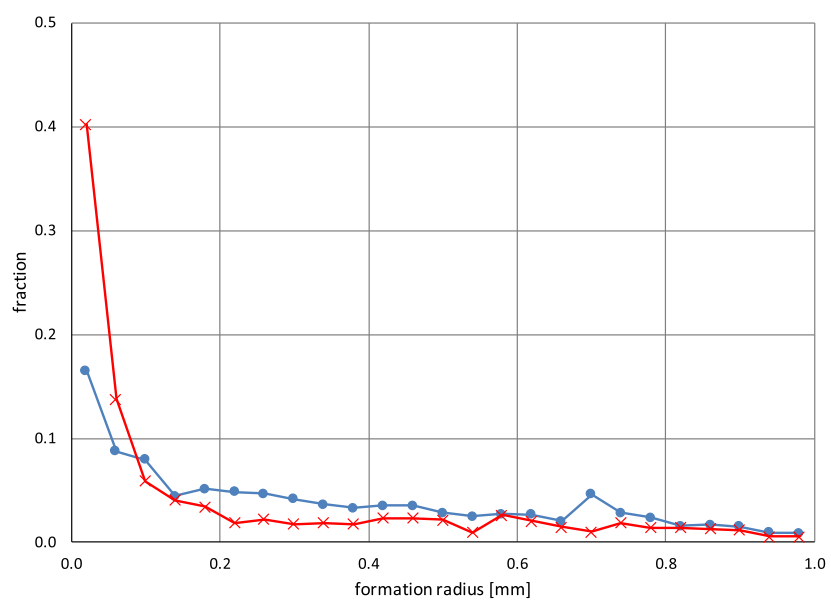

Figure 6. The fraction of stable $\overline{\mathrm{H}}$ versus the distance from the trap axis where they were formed, following $\bar{p}$ injection at $\rho_{0}=0$, at densities of $10^{14} \mathrm{~m}^{-3}$ (blue circles) and $10^{15} \mathrm{~m}^{-3}$ (red crosses) for $T_{\mathrm{e}}=10 \mathrm{~K}$ and $B=1 \mathrm{~T}$.

observed at 5.4 $\mathrm{T}$ (when compared to $1 \mathrm{~T}$ ) in figure 4 can be attributed to the much reduced effect of $\Delta v_{\mathrm{D}}$ at this field. The fall in the overall $f_{\overline{\mathrm{H}}}$ at high densities shown in figure 1 can also be attributed to the differential positron/antiproton drift: again, the effect is reduced at the higher magnetic field.

This interpretation is supported by the results in figure 6, where we have isolated the starting radial position in the $\mathrm{e}^{+}$ plasma which led to the formation of the stable $\bar{H}^{5}$. The figure shows the distributions of stable $\overline{\mathrm{H}}$ starting positions for $T_{\mathrm{e}}=10 \mathrm{~K}, B=1 \mathrm{~T}$ and at $n_{\mathrm{e}}=10^{14}$ and $10^{15} \mathrm{~m}^{-3}$, which coincides with the density region (see figure 3 ) where the $E_{\mathrm{B}}$ distributions begin to change form. Although it is possible that $\overline{\mathrm{H}}$ is formed anywhere in the plasma, there is a dramatic shift to formation close to $\rho=0$ as $n_{\mathrm{e}}$ is raised, with around $40 \%$ originating within $0.04 \mathrm{~mm}$ of the axis at $10^{15} \mathrm{~m}^{-3}$. This has fallen to around $16 \%$ at the lower density shown in the figure. According to our interpretation, formation of stable $\overline{\mathrm{H}}$ is suppressed at large $\rho$ and high density because of the mechanism discussed above.

\section{Conclusion}

We have provided a detailed account of the dependence of the binding energies of $\overline{\mathrm{H}}$ atoms formed under conditions similar to those encountered in recent antimatter experimentation, and in particular simulation data have been presented over typical ranges of $\mathrm{e}^{+}$plasma density and temperature.

The picture that has emerged is complex, and involves qualitative and quantitative changes of behaviour as $n_{\mathrm{e}}$ is increased beyond about $10^{14} \mathrm{~m}^{-3}$, and in the $T_{\mathrm{e}}$ range from $5-30 \mathrm{~K}$ and for the two magnetic fields ( 1 and $5.4 \mathrm{~T}$ ) investigated. These

\footnotetext{
${ }^{5}$ Note the distinction between this entity and the $\bar{p}$ injection radius, $\rho_{0}$. Recall from section 2 that for most of the work $\bar{p}$ trajectories were initialised on the axis of the system (i.e., at $\rho_{0}=0$ ), whereupon they drift radially, largely due to cycles of $\overline{\mathrm{H}}$ formation and destruction, before they possibly form stable $\overline{\mathrm{H}}$. Thus, the $\overline{\mathrm{H}}$ starting radius may extend from $\rho=0$ out to the plasma edge of $1 \mathrm{~mm}$ considered here.
}

have been linked to the role of $\mathrm{e}^{+}$collisions and their effect on the weakly bound states of $\overline{\mathrm{H}}$ formed via reaction 1 in such low temperature, high density $\mathrm{e}^{+}$plasmas. A defining feature is the emergence of antihydrogen states of higher binding energy, broadly speaking as $n_{\mathrm{e}}$ is raised and $T_{\mathrm{e}}$ is lowered. The high $E_{\mathrm{B}}$ tails of the resulting distributions exhibit power-law behaviour similar to that found previously $[25,31]$. Our work has expanded considerably on these earlier studies, revealing a much richer behaviour by using a simulation scenario of direct relevance to contemporary $\overline{\mathrm{H}}$ experimentation.

In particular we have shown that while the overall number of $\bar{H}$ is somewhat reduced at large densities, the binding energies of the $\overline{\mathrm{H}}$ produced are on average much larger. However, this effect is only present for the fraction of antiprotons injected close to the trap axis $\left(\rho_{0} \lesssim 0.1 \mathrm{~mm}\right)$ for the simulated conditions. The importance of this parameter has not been given much attention in previous works.

\section{Acknowledgments}

MC thanks the EPSRC (UK) for support of his antihydrogen work and SJ wishes to acknowledge the Swedish Research Council (VR) for financial support (Grant No. 2017-03822), and the High Performance Computing Centre North (HPC2N) for computing resources.

\section{ORCID iDs}

S Jonsell (D) https://orcid.org/0000-0003-4969-1714

M Charlton (D) https://orcid.org/0000-0002-9754-1932

\section{References}

[1] Bertsche W A, Butler E, Charlton M and Madsen N 2015 J. Phys. B: At. Mol. Opt. Phys. 48232001

[2] Andresen G B et al (ALPHA Collaboration) 2010 Nature 468 673

[3] Andresen G B et al (ALPHA Collaboration) 2011 Phys. Lett. B 69595

[4] Andresen G B et al (ALPHA Collaboration) 2011 Nat. Phys. 7 558

[5] Gabrielse G et al (ATRAP Collaboration) 2012 Phys. Rev. Lett. 108113002

[6] Ahmadi M et al (ALPHA Collaboration) 2017 Nat. Commun. 8 681

[7] Amole C et al (ALPHA Collaboration) 2012 Nature 483439

[8] Amole C et al (ALPHA Collaboration) 2014 Nat. Commun. 5 3955

[9] Ahmadi M et al (ALPHA Collaboration) 2016 Nature 529373

[10] Ahmadi M et al (ALPHA Collaboration) 2017 Nature 54866

[11] Ahmadi M et al (ALPHA Collaboration) 2017 Nature 541 506

[12] Ahmadi M et al (ALPHA Collaboration) 2018 Nature 55771

[13] Ahmadi M et al (ALPHA Collaboration) 2018 Nature 561211

[14] Ahmadi M et al (ALPHA Collaboration) 2020 Nature 578 375

[15] Amoretti M et al (ATHENA Collaboration) 2002 Nature 419 456

[16] Gabrielse G et al (ATRAP Collaboration) 2002 Phys. Rev. Lett. 89213401

[17] Robicheaux F 2008 J. Phys. B: At. Mol. Opt. Phys. 41192001 
[18] Jonsell S, van der Werf D P, Charlton M and Robicheaux F 2009 J. Phys. B: At. Mol. Opt. Phys. 42215002

[19] Jonsell S, Charlton M and van der Werf D P 2016 J. Phys. B: At. Mol. Opt. Phys. 49134004

[20] Jonsell S and Charlton M 2018 New J. Phys. 20043049

[21] Amole C et al(ALPHA Collaboration) 2014 Nucl. Instrum. Methods A 735319

[22] Holzscheiter M H, Charlton M and Nieto M M 2004 Phys. Rep. 4021

[23] Gabrielse G et al (ATRAP Collaboration) 2002 Phys. Rev. Lett. 89233401

[24] Vrinceanu D et al 2004 Phys. Rev. Lett. 92133402

[25] Pohl T, Sadeghpour H R and Gabrielse G 2006 Phys. Rev. Lett. 97143401

[26] Jelenković B M, Newbury A S, Bollinger J J, Itano W M and Mitchell T B 2003 Phys. Rev. A 67063406

[27] Madsen N, Robicheaux F and Jonsell S 2014 New J. Phys. 16 063046

[28] Sameed M, Maxwell D and Madsen N 2020 New J. Phys. 22 013009
[29] Jones J, Madsen N, Maxwell D T and Peszka J 2020 private communication

[30] Gabrielse G et al (ATRAP Collaboration) 2001 Phys. Lett. B 5071

[31] Bass E M and Dubin D H E 2009 Phys. Plasmas 16012101

[32] Radics B, Murtagh D J, Yamazaki Y and Robicheaux F 2014 Phys. Rev. A 90032704

[33] Radics B and Yamazaki Y 2016 J. Phys. B: At. Mol. Opt. Phys. 49064007

[34] Gallagher T F 2005 Rydberg Atoms (Cambridge Monographs on Atomic, Molecular and Chemical Physics) (Cambridge: Cambridge University Press)

[35] Müller A and Wolf A 1997 Hyperfine Interact. 109233

[36] Byron S, Stabler R C and Bortz P I 1962 Phys. Rev. Lett. 8 376

[37] Mansbach P and Keck J 1969 Phys. Rev. 181275

[38] Stevefelt J, Boulmer J and Delpech J-F 1975 Phys. Rev. A 12 1246

[39] Glinsky M E and O’Neil T M 1991 Phys. Fluids B 31279 